\title{
Seasonal Frequency in Esophageal Atresia/Tracheo-Esophageal Fistula: Is there an Environmental Etiology?
}

\author{
Christopher Bode, Adesoji Ademuyiwa, Sylvester Ikhisemojie \\ Pediatric Surgery Unit, Department of Surgery, College of Medicine of the University of Lagos/Lagos University \\ Teaching Hospital, Lagos, Nigeria \\ E-mail: cobode@yahoo.com
}

Received May 13, 2011; revised July 13, 2011; accepted August 9, 2011

\begin{abstract}
Background: Esophageal Atresia and Tracheo-Esophageal Fistula (EA/TEF) occurs sporadically and its etiology is poorly understood. We observed six cases of EA/TEF within three weeks of October 2007 at the Lagos University Teaching Hospital (LUTH), Nigeria. Aims: To analyze the monthly trends of presentation of EA/TEF at the LUTH from 2002-7, test the hypothesis that the October figures were not due to chance and correlate data with known local disease patterns. Setting: Lagos University Teaching Hospital, Nigeria. Patients, Materials, Method: A retrospective analysis of all EA/TEFs managed at the LUTH within stated period. Results: 25 babies with EA/TEF presented over the period. 10 (40\%) were seen in the month of October, 5 (20\%) occurred in May while the rest were spread over the rest of the months. Chi-square analysis confirmed that the EA/TEF cases occurred significantly more frequently in October $(\mathrm{P}<0.001)$ and this observation was unlikely due to chance. Conclusions: There seems to be a seasonal variation in the occurrence of EA/TEF in Lagos, with a significantly higher frequency in the month of October. $96 \%$ of mothers of babies with EA/TEF lived in low socioeconomic parts of Lagos. This study highlights the possibility of local viral etiology of EA/TEF.
\end{abstract}

Keywords: Tracheo-Esophageal Fistula, Seasonal Etiology

\section{Introduction}

The etiology of oesophageal atresia with tracheo-esophageal fistula (EA/TEF) has been a subject of intensive investigations over the years but little if anything is known about specific causative agents in humans [1]. No definite seasonal variations or patterns have been demonstrated for this condition [2]. Its occurrence is sometimes sporadic but these are confined to those with rare familial genetic dispositions [3]. The unusual presentation of six new cases of EA/TEF to our Unit within three weeks of October 2007 at the Lagos University Teaching Hospital therefore prompted a search for possible environmental factors that could have triggered the cluster. We sought to analyze the trend and test the hypothesis that the October 2007 figures were not due to chance and correlate our data with known local disease patterns.

\section{Materials/Methods}

Records of all cases of esophageal atresia managed by the Pediatric Surgical Unit at the LUTH between January 2002 and December 2007 were retrospectively compiled and analyzed for date of presentation, age at presentation, maternal age, tribe, maternal socio-economic locale, occupation, maternal last menstrual period, history of occupational exposure to chemical teratogens, and firsttrimester maternal illness.

Addresses of parents were categorized as affluent, middle-class or low based on the demographic spread of the Lagos metropolis. All the data were recorded onto a protocol and analyzed using Microsoft Excel 2007. The observed monthly frequency was subjected to Chi-square analysis to assess whether the difference between the observed and expected distributions could be attributed 
to chance and unless otherwise stated, $\mathrm{P}<0.05$ was considered statistically significant.

\section{Results}

25 babies with EA/TEF were seen within the study period. There were 14 (56\%) males and 11 (44\%) females, giving a male:female ratio of 1.2:1. Their ages ranged from 10 hours to 25 days with a mean age of $8.8 \pm 5.2$ days at presentation. The babies were all full-term, with a mean birth weight of $2.6 \pm 0.4 \mathrm{~kg}$ (range $2.1-3.7 \mathrm{~kg}$ ). Seventeen (68\%) of these babies were first borns.

Maternal age ranged from 20 - 31 years, with a mean of $24.5 \pm 3.3$ years. All the 25 mothers were Nigerian. There was no preponderance of any ethnic group or occupation among the mothers. None of the mothers gave any history of exposure to heavy metals or known teratogens. None of the babies was prenatally diagnosed as having EA/TEF. Twenty four mothers (96\%) lived in low socio-economic parts of Lagos.

\subsection{Monthly Frequency of EA/TEF Occurence}

Table 1 shows the monthly frequency of EA/TEF over the five year period under review. The months of January, February, July and September recorded one case each while 2 cases were recorded for each of March, June and August. Five cases were seen in May while 10 were recorded in October. April, November and December recorded none.

The expected monthly distribution was 25/12 $=2.08$ cases per month, assuming that all cases occurred with equal frequency over a twelve month period. This was subjected to the Chi-square analysis, to examine whether the difference between the observed and expected distributions was attributable to chance. A low P-value of < 0.001 obtained did not support the null hypothesis that there was no difference between the observed and expected distributions. Rather, it supported the alternative hypothesis that the difference observed was unlikely to

Table 1. Observed monthly trends 2002-7.

\begin{tabular}{cc}
\hline Month & Frequency \\
\hline Jan & 1 \\
Feb & 1 \\
March & 2 \\
April & 0 \\
May & 5 \\
June & 2 \\
July & 1 \\
August & 2 \\
Sept & 1 \\
Oct & 10 \\
Nov & 0 \\
Dec & 0 \\
Total & 25
\end{tabular}

be due to chance, and thus, the occurrence of a seasonal variation in the occurrence of EA/TEF was real.

\subsection{Maternal Last Menstrual Period}

The two peak periods of May and October raised the specter of possible seasonal environmental teratogenic events early in the first trimester. We therefore sought the last menstrual periods of the mothers and this showed that the babies born in May were conceived in August while the October ones were conceived in January.

\section{Discussion}

This is the first known report of a seasonal occurrence of EA/TEF from Africa to the best of our knowledge. A wide search of the local and international literature revealed no similar occurrence. It is interesting to know that the babies born in October and May were conceived in January and August respectfully. August, January and April are months when seasons change in Lagos. These three periods constitute peak seasons of viral loads in Lagos (during which there occurs widespread respiratory and gastro-enteric infections) and we had earlier shown these periods of seasonal changes to be associated with the highest frequency of idiopathic intussusceptions in the Lagos metropolis [4]. Our previous studies had reported adenoviruses in $40 \%$ of idiopathic intussusceptions [5]. Similarly, Olaleye et al. [6] showed that the peak incidence of influenza infections occurred between April and September and during the harmattan season in November among children in Ibadan, a large Nigerian city $130 \mathrm{~km}$ northeast of Lagos. These periods coincide roughly with the occurrence of idiopathic intussusceptions and therefore of the exposure to viruses that may likely be causative agents for EA/TEF. This notion is underscored by the earlier suggestion that viral teratogenicity in EA/TEF constitutes part of atresias involving the biliary, anorectal and esophageal tracts [7]. Additional support for our hypothesis comes from the studies of Ozimek et al. [8] who observed a clustering and cyclic variation in the occurrence of EA/TEF similar to that of infectious hepatitis in North Carolina.

We thus hypothesize that a possible exposure of pregnant mothers to respiratory and gastrointestinal viral agents in the first trimester during these periods interfered in some yet unknown way with the esophageal embryogenesis to cause the EA/TEF. The long interval between teratogenicity and postnatal diagnosis would seem to separate these events by several months and possibly distract the attention of investigators from focusing on that phase of development. Future studies should aim at identifying viral markers in babies born 
under similar conditions as well as in their mothers.

\subsection{Limitations}

This was a retrospective observational study occasioned by the occurrence of a cluster of EA/TEF noticed in October 2007. The gestational gap between the intrauterine teratogenic insult to the esophagus and the postnatal presentation of EA/TEF limited the possible elucidation of viral fingerprints in the babies and their mothers. Our institution had difficulties with theatre facilities which were under repairs in 2005 - 2006 and consequently, experienced a reduction in the number of referrals to our Unit during this period.

\subsection{Conclusions}

Our study suggests that there is a seasonal variation in the occurrence of EA/TEF in Lagos with a significantly higher frequency in the month of October. 96\% of mothers of babies with EA/TEF lived in low socioeconomic parts of Lagos. Future studies should be directed to identifying possible viral teratogens in this group of newborns.

\section{References}

[1] J. F. Felix, M. F. van Dooren, M. Klaasens, W. C. Hop, C. P. Torfs, et al., "Environmental Factors in the Etiology of Esophageal Atresia and Congenital Diaphragmatic Hernia: Results of a Case-Controlled Study," Birth Defects Re- search. Part A, Clinical and Molecular Teratology, Vol. 82, No. 2, 2008, pp. 98-105. doi:10.1002/bdra.20423

[2] C. Fraser, P. A. Baird and A. D. Sadovnick, "A Comparison of Incidence Trends for Esophageal Atresia and Tracheoesophageal Fistula, and Infectious Disease," Teratology, Vol. 36, No. 3, 1987, pp. 363-369.

[3] M. Van Staey, S. De Bies, M. T. Matton and J. De Roose, "Familial Congenital Esophageal Atresia. Personal Case Report and Review of the Literature," Human Genetics, Vol. 66, No. 2-3, 1984, pp. 260-266.

[4] C. O. Bode, "Presentation and Management Outcome of Intussusceptions in Lagos: A Prospective Study,” African Journal of Paediatric Surgery, Vol. 5, No. 1, 2008, pp. 24-28.

[5] C. O. Bode and S. A. Omilabu, "Viral Isolates of Intussusceptions in Nigerian Infants," South African Journal of Surgery, Vol. 40, No. 2, 2002, pp. 57-58.

[6] O. D. Olaleye, S. A. Omilabu, A. O. Olabode and A. H. Fagbami, "Serological Evidence for Influenza Virus Activity in Nigeria (1985-1987),” Virologie, Vol. 40, No. 1, 1989, pp. 11-17.

[7] A. Dessanti, G. Massarelli, M. T. Piga, A. Porcu and G. Dettori, "Biliary, Anorectal and Esophageal Atresia: A New Entity?” Tohoku Journal of Experimental Medicine, Vol. 181, No. 1, 1997, pp. 49-55. doi:10.1620/tjem.181.49

[8] C. D. Ozimeck, R. C. Grimson and A. S. Aylsworth, “AN Epidemiologic Study of Tracheoesophageal Fistula and Esophageal Atresia in North Carolina,” Teratology, Vol. 25, No. 1, 1982, pp. 53-59. 